\title{
LA-UR-21-29166
}

Approved for public release; distribution is unlimited.

Title:

Author(s):

Intended for:

Issued:
Physics of the Serrano Sn Transport Package

Budge, Kent Grimmett

Report

2021-09-16 
Disclaimer:

Los Alamos National Laboratory, an affirmative action/equal opportunity employer, is operated by Triad National Security, LLC for the National Nuclear Security Administration of U.S. Department of Energy under contract 89233218CNA000001. By approving this article, the publisher recognizes that the U.S. Government retains nonexclusive, royalty-free license to publish or reproduce the published form of this contribution, or to allow others to do so, for U.S. Government purposes. Los Alamos National Laboratory requests that the publisher identify this article as work performed under the auspices of the U.S. Department of Energy. Los Alamos National Laboratory strongly supports academic freedom and a researcher's right to publish; as an institution, however, the Laboratory does not endorse the viewpoint of a publication or guarantee its technical correctness. 


\section{Physics of the Serrano Sn Transport Package}

Kent G. Budge

LA-UR-21-xxxxx 16 September 2021 


\section{Variables Used in This Report}

\begin{tabular}{|c|c|c|}
\hline Variable & Units & Definition \\
\hline$x$ & $\mathrm{~cm}$ & Position \\
\hline$t$ & $\mathrm{~s}$ & Time \\
\hline$\vec{u}$ & $\mathrm{~cm} / \mathrm{s}$ & Velocity \\
\hline$\rho$ & $\mathrm{g} / \mathrm{cm}^{3}$ & Mass density \\
\hline$\hat{n}$ & 1 & Ordinate vector \\
\hline$v$ & $\mathrm{~Hz}$ & Photon frequency in laboratory frame \\
\hline$v_{0}$ & $\mathrm{~Hz}$ & Photon frequency in comoving frame \\
\hline$I_{v}$ & $\mathrm{erg} / \mathrm{cm}^{2} / \mathrm{s} / \mathrm{Hz} / \mathrm{sr}$ & Specific intensity \\
\hline$E_{v}$ & $\mathrm{erg} / \mathrm{cm}^{3} / \mathrm{Hz}$ & $\begin{array}{l}\text { Monochromatic radiation energy } \\
\text { density }\end{array}$ \\
\hline $\overrightarrow{F_{v}}$ & $\mathrm{erg} / \mathrm{cm}^{2} / \mathrm{s} / \mathrm{Hz}$ & Monochromatic radiation energy flux \\
\hline $\boldsymbol{P}_{v}$ & $\mathrm{erg} / \mathrm{cm}^{3} / \mathrm{Hz}$ & $\begin{array}{l}\text { Monochromatic radiation pressure } \\
\text { tensor }\end{array}$ \\
\hline$B_{v}$ & $\mathrm{erg} / \mathrm{cm}^{2} / \mathrm{s} / \mathrm{Hz} / \mathrm{sr}$ & Planck black-body function \\
\hline$E$ & $\mathrm{erg} / \mathrm{cm}^{3}$ & Total radiation energy density \\
\hline$\vec{F}$ & $\mathrm{erg} / \mathrm{cm}^{2} / \mathrm{s}$ & Total radiation energy flux \\
\hline$B$ & $\mathrm{erg} / \mathrm{cm}^{2} / \mathrm{s} / \mathrm{sr}$ & Frequency-integrated Planck function \\
\hline$T_{e}$ & $\mathrm{~K}$ & Electron temperature \\
\hline$T_{I}$ & $\mathrm{~K}$ & Ion temperature \\
\hline$\sigma_{a}$ & $1 / \mathrm{cm}$ & Absorption cross-section \\
\hline$\sigma_{s}$ & $1 / \mathrm{cm}$ & Scattering cross-section \\
\hline$\sigma_{t}$ & $1 / \mathrm{cm}$ & Total cross-section \\
\hline$\phi$ & $\mathrm{erg} / \mathrm{g}$ & Gravitational potential \\
\hline$p_{e}$ & dyne $/ \mathrm{cm}^{2}$ & Electron pressure \\
\hline$p_{I}$ & dyne $/ \mathrm{cm}^{2}$ & Ion pressure \\
\hline$U_{e}$ & $\mathrm{erg} / \mathrm{g}$ & $\begin{array}{l}\text { Specific internal energy from } \\
\text { electrons }\end{array}$ \\
\hline$U_{I}$ & $\mathrm{erg} / \mathrm{g}$ & Specific internal energy from ions \\
\hline$\lambda_{C}$ & $\mathrm{erg} / \mathrm{cm} / \mathrm{s} / \mathrm{K}$ & Thermal conduction coefficient \\
\hline$\varepsilon_{e}$ & $\mathrm{erg} / \mathrm{s} / \mathrm{g}$ & $\begin{array}{l}\text { Electron specific energy deposition } \\
\text { rate }\end{array}$ \\
\hline$\varepsilon_{I}$ & $\mathrm{erg} / \mathrm{s} / \mathrm{g}$ & Ion specific energy deposition rate \\
\hline 1 & 1 & The identity tensor \\
\hline
\end{tabular}

\section{Introduction}

Serrano is a software package for simulating photon transport in moving material. It presently supports 1-D, unstructured 2-D for a wide variety of element types, and 3-D for tetrahedra and hexahedra. The package is designed to be a component of larger codes, such as radiation hydrodynamics codes. Only the radiation transport equations are solved by Serrano itself. 
Serrano solves the photon transport equation in the Sn approximation in the laboratory frame. Only those material motion corrections required to conserve energy and momentum and to preserve the diffusion limit and the correct equilibrium state are included.

\section{Governing Equations}

\subsection{Variables}

The Boltzmann equation for photon transport is (Lowrie \& Morel, 1998)

$$
\frac{1}{c} \frac{\partial I_{v}}{\partial t}+\hat{n} \cdot \nabla I_{v}+\frac{v_{0}}{v} \sigma_{t, v_{0}} I_{v}=\left(\frac{v}{v_{0}}\right)^{2} \sigma_{a, v_{0}} B_{v_{0}}\left(T_{e}\right)+\left(\frac{v}{v_{0}}\right)^{2} \frac{\sigma_{s, v_{0}}}{4 \pi} \int \frac{v_{0}}{v^{\prime}} I_{v^{\prime}} d \hat{n}^{\prime}
$$

where

$$
v_{0}=v \gamma\left(1-\frac{\hat{n} \cdot \vec{u}}{c}\right)
$$

$$
\gamma=\frac{1}{\sqrt{1-\frac{u^{2}}{c^{2}}}}
$$

$$
v^{\prime}=v \frac{1-\frac{\hat{n} \cdot \vec{u}}{c}}{1-\frac{\widehat{n}^{\prime} \cdot \vec{u}}{c}}
$$

This assumes an isotropic and coherent scattering opacity. In reality, electron scattering is neither isotropic nor coherent, although in the low-energy limit (Thompson scattering) it is very nearly coherent and its angular dependence is approximately a dipole scattering function, which behaves much like an isotropic scattering function in the diffusion limit. Future versions of Serrano will use the correct dipole scattering function, but for now, the assumption of isotropic scattering allows great simplification.

In the Lagrangian frame, the Boltzmann equation becomes

$$
\frac{\rho}{c} \frac{D}{D t}\left(\frac{I_{v}}{\rho}\right)-\nabla \cdot\left(\frac{\vec{u}}{c} I_{v}\right)+\hat{n} \cdot \nabla I_{v}+\frac{v_{0}}{v} \sigma_{t, v_{0}} I_{v}=\left(\frac{v}{v_{0}}\right)^{2} \sigma_{a, v_{0}} B_{v_{0}}\left(T_{e}\right)+\left(\frac{v}{v_{0}}\right)^{2} \frac{\sigma_{s, v_{0}}}{4 \pi} \int \frac{v_{0}}{v^{\prime}} I_{v^{\prime}} d \hat{n}^{\prime}
$$

Followng Lowrie and Morel, we omit all terms of order $u / c$ or greater that are not necessary to preserve the correct asymptotic diffusion limit in a moving medium. We are careful to note that the radiation quantities are expressed in the laboratory frame while the cross-sections, density, and temperature are comoving values. This yields 


$$
\frac{\rho}{c} \frac{D}{D t}\left(\frac{I_{v}}{\rho}\right)-\nabla \cdot\left(\frac{\vec{u}}{c} I_{v}\right)+\hat{n} \cdot \nabla I_{v}+\sigma_{t, v} I_{v}=\sigma_{a, v} B_{v}\left(T_{e}\right)+\frac{c}{4 \pi} \sigma_{s, v} E_{v}+\frac{1}{4 \pi c}\left(C_{0, v}+3 \vec{C}_{1, v} \cdot \hat{n}\right)
$$

where

$$
C_{0, v}=\left(\sigma_{a, v}-\sigma_{s, v}\right) \vec{u} \cdot\left(\vec{F}_{v}-\frac{4}{3} \vec{u} E_{v}\right)
$$

$$
\vec{C}_{1, v}=\frac{4}{3} \sigma_{t, v} c E_{v} \vec{u}
$$

$$
E_{v}=\int I_{v} d \hat{n}
$$

$$
\vec{F}_{v}=\int I_{v} \hat{n} d \hat{n}
$$

We assume that Serrano is called by a host code that solves the hydrodynamics equations:

$$
\rho \frac{D}{D t}\left(\frac{1}{\rho}\right)=\nabla \cdot \vec{u}
$$

$$
\rho\left(\frac{D \vec{u}}{D t}+\nabla \phi\right)=-\nabla p+\frac{1}{c} \int \sigma_{t}\left(\vec{F}_{v}-\frac{4}{3} \vec{u} E_{v}\right) d v
$$

$$
\begin{aligned}
& \quad \rho \frac{D U_{e}}{D t}=-p_{e} \nabla \cdot \vec{u}+\nabla \cdot\left(\lambda_{c} \nabla \cdot T_{e}\right)+\alpha\left(T_{I}-T_{e}\right)+\rho \varepsilon_{e}+\int \sigma_{a, v}\left(c\left(E_{v}-2 \frac{\vec{u}}{c^{2}} \cdot\left(\vec{F}_{v}-\frac{4}{3} \vec{u} E_{v}\right)\right)-\right. \\
& \left.4 \pi B_{v}\left(T_{e}\right)\right) d v
\end{aligned}
$$

$$
\rho \frac{D U_{I}}{D t}=-p_{I} \nabla \cdot \vec{u}+\left(T_{e}-T_{I}\right)+\rho \varepsilon_{I}
$$

The exact form of the equations solved by the host code may differ from these, but appropriate modifications can be made to ensure conservative coupling between Serrano and its host.

These hydrodynamics equations assume that the ion and electron distributions are Maxwellian, but at different ion and electron temperatures. This is justified because the large mass disparity between electrons and ions $\left(m_{I}=1860 \mathrm{Am}_{e}\right)$ means that electron-ion collisions will not exchange energy efficiently. Thus physical processes, such as radiation absorption, that couple to only one component of the plasma will tend to pull the two components out of equilibrium with each other. 


\subsection{Conservation of Energy and Momentum}

The zeroth and first moments of the transport equation are

$$
\begin{aligned}
& \quad \frac{\rho}{c} \frac{D}{D t}\left(\frac{E_{v}}{\rho}\right)-\nabla \cdot\left(\vec{u} E_{v}+\vec{F}_{v}\right)+=\sigma_{a, v}\left(4 \pi B_{v}\left(T_{e}\right)-c\left(E_{v}-2 \frac{\vec{u}}{c^{2}} \cdot\left(\vec{F}_{v}-\frac{4}{3} \vec{u} E_{v}\right)\right)\right)-\sigma_{t, v} \frac{\vec{u}}{c} \\
& \left(\vec{F}_{v}-\frac{4}{3} \vec{u} E_{v}\right)
\end{aligned}
$$

$$
\rho \frac{D}{D t}\left(\frac{\vec{F}_{v}}{c^{2} \rho}\right)-\nabla \cdot\left(\frac{\vec{u}}{c^{2}} \otimes \vec{F}_{v}+\boldsymbol{P}_{v}\right)=\frac{1}{c} \sigma_{t, v}\left(\vec{F}_{v}-\frac{4}{3} \vec{u} E_{v}\right)
$$

where

$$
\boldsymbol{P}_{\boldsymbol{v}}=\frac{1}{c} \int_{4 \pi} I_{v} \hat{n} \otimes \hat{n} d \hat{n}
$$

A comparison of these equations with the host hydrodynamics equations (Equations 11 to 14) shows that Serrano and its host will conserve energy and momentum if the couplings to the host code are carried out correctly.

\section{Temporal Discretization}

There are numerous temporal discretizations that one might employ to solve the governing equations. Serrano provides a flexible enough interface to support more than one time discretization. We will illustrate with the example of a predictor-corrector time discretization.

In all cases, Serrano assumes an operator split, and solves the radiation transport portio of the split using a semi-implicit time discretization. Serrano relies on the host code to perform any remap of the radiation energy density that is required. This is best accomplished by including radiation energy per unit mass as a variable in the host code database, and treating it exactly like specific energy for purposes of remapping.

\subsection{Predictor-Correct Time Discretization}

This is a second-order explicit time discretization in which all quantities have the same time centering.

The host hydrocode should perform the following steps:

\section{Remap:}

The host code first performs any mesh relaxation and remapping of variables. This step is highly hostcode specific. To satisfy energy conservation, the specific intensity $\frac{I_{v, i}}{\rho_{i}}$ should be advected like the specific energies $U_{i}$. 


\section{Predict new velocities:}

$$
\vec{u}_{i+1}^{*}=\vec{u}_{i}-\left(\frac{\nabla p_{i}-\frac{1}{c} \int \sigma_{t, v, i}\left(\vec{F}_{v, i}-\frac{4}{3} \vec{u}_{i} E_{v, i}\right) d v}{\varrho_{i}}+\nabla \phi_{i}\right) \Delta t
$$

where $i$ is a time level and a superscript asterisk indicates a predicted value from a step in an operator split. The cost code is expected to include a radiation acceleration term $\frac{1}{c} \int \sigma_{t, v, i}\left(\vec{F}_{v, i}-\frac{4}{3} \vec{u}_{i} E_{v, i}\right) d v$ supplied by Serrano. For a staggered-grid hydrodynamics spatial discretization, this will take the form of a radiation force applied to each node.

$$
\vec{x}_{i+1}^{*}=\vec{x}_{i}+\frac{\vec{u}_{i}+\vec{u}_{i+1}^{*}}{2} \Delta t
$$

\section{Predict new state:}

$$
\begin{aligned}
& \frac{\rho_{i+1}^{*}}{J\left(\vec{x}_{i+1}^{*}\right)}=\frac{\rho_{i}}{J(\vec{x})} \\
& U_{e, i+1}^{*}=U_{e, i}+\left(\frac{1}{2}\left(\vec{u}_{i+1}^{*}+\vec{u}_{i}\right) \cdot \frac{\nabla p_{e, i}}{\rho_{i}}+\varepsilon_{e, i}\right) \Delta t \\
& U_{I, i+1}^{*}=U_{I, i}+\left(\frac{1}{2}\left(\vec{u}_{i+1}^{*}+\vec{u}_{i}\right) \cdot \frac{\nabla p_{I, i}}{\rho_{i}}+\varepsilon_{I, i}\right) \Delta t \\
& \frac{I_{v, i+1}^{*}}{\rho_{i+1}^{*}}=\frac{I_{v, i}}{\rho_{i}}-\frac{\sigma_{t, v, i}}{2 \rho_{i} c} I_{\nu, i}\left(\vec{u}_{i+1}^{*}+\vec{u}_{i}\right) \cdot\left(\hat{n}-\frac{4}{3 c} \vec{u}_{i}\right) \Delta t
\end{aligned}
$$

where $J$ is the Jacobian describing the deformation of the original Lagrangian mesh. The Jacobian typically corresponds to some Lagrangian control volume such as a cell volume. The host code is again expected to pass the starting and ending velocities $\vec{u}_{i}$ and $\vec{u}_{i+1}^{*}$ to Serrano so that Serrano can update the radiation specific intensity $I_{v}$ to reflect work done by the radiation force.

\section{Correct the velocity prediction:}

$$
\vec{u}_{i+1}=\vec{u}_{i}-\left(\frac{\nabla\left(p_{i}+p_{i+1}^{*}\right)-\frac{1}{2 c} \int \sigma_{t, v, i}\left(\vec{F}_{v, i}-\frac{4}{3} \vec{u}_{i} E_{v, i}\right) d v}{\varrho_{i}+\varrho_{i+1}^{*}}+\frac{\nabla\left(\phi_{i}+\phi_{i+1}^{*}\right)}{2}\right) \Delta t
$$

The spatial discretization usually replaces the density with lumped mass, which is a Lagrangian constant, so the density average appearing in the equation is strictly a formality. The radiation force is normally fixed at its value at the start of the time step. Serrano has experimental options under development that relax this operator split.

\section{Correct the coordinate prediction:}

$$
\vec{x}_{i+1}=\vec{x}_{i}+\frac{\vec{u}_{i}+\vec{u}_{i+1}}{2} \Delta t
$$

\section{Correct the state prediction:}

$$
\frac{\rho_{i+1}}{J\left(\vec{x}_{i+1}\right)}=\frac{\rho_{i}}{J(\vec{x})}
$$




$$
\begin{aligned}
& U_{e, i+1}^{* *}=U_{e, i}+\left(\frac{1}{2}\left(\vec{u}_{i+1}+\vec{u}_{i}\right) \cdot \frac{\nabla\left(p_{e, i}+p_{e, i+1}^{*}\right)}{\rho_{i}}+\frac{1}{2}\left(\varepsilon_{e, i}+\varepsilon_{e, i+1}^{*}\right)\right) \Delta t \\
& U_{I, i+1}^{* *}=U_{I, i}+\left(\frac{1}{2}\left(\vec{u}_{i+1}+\vec{u}_{i}\right) \cdot \frac{\nabla\left(p_{I, i}+p_{I, i+1}^{*}\right)}{\rho_{i}}+\frac{1}{2}\left(\varepsilon_{I, i}+\varepsilon_{I, i+1}^{*}\right)\right) \Delta t \\
& \frac{I_{v, i+1}^{* *}}{\rho_{i}}=\frac{I_{v, i}}{\rho_{i}}-\frac{1}{2 c} \sigma_{t, v, i} I_{v, i}\left(\frac{\vec{u}_{i+1}+\vec{u}_{i}}{\varrho_{i}+\varrho_{i+1}^{*}}\right) \cdot\left(\hat{n}-\frac{4}{3 c} \vec{u}_{i}\right) \Delta t
\end{aligned}
$$

Equation 29 accounts for both radiation work and changes in the control volume. It ensures conservation of energy. Note also that there is no $I_{v, i+1}^{*}$ in this formulation, since the radiation force is not adjusted following the prediction step.

The remaining calculations are performed by Serrano, which solves the radiation transport and coupled material energy equations

$$
\begin{aligned}
& \rho \frac{U_{e, i+1}-U_{e, i+1}^{* *}}{\Delta t}=\alpha\left(T_{I, i+1}^{* * *}-T_{e, i+1}^{* * *}\right)+\int \sigma_{a, v, i}\left(c E_{v, i+1}-4 \pi B\left(T_{e, i+1}^{* * *}\right)\right) d v \\
& \rho \frac{U_{I, i+1}-U_{I, i+1}^{* *}}{\Delta t}=\alpha\left(T_{e, i+1}^{* * *}-T_{I, i+1}^{* * *}\right) \\
& \frac{I_{v, i+1}-I_{v, i+1}^{* *}}{c \Delta t}+\hat{n} \cdot \nabla I_{v, i+1}+\sigma_{t, s, i} I_{v, i+1}=\sigma_{v, a, i} B_{v}\left(T_{e, i+1}^{* * *}\right)+\frac{c}{4 \pi} \sigma_{v, s, i} E_{v, i+1}
\end{aligned}
$$

where

$$
\begin{aligned}
& T_{e, i+1}^{* * *}=T_{e, i+1}^{* *}+\frac{U_{e, i+1}-U_{e, i+1}^{* *}}{C_{V, e}} \\
& T_{I, i+1}^{* * *}=T_{I, i+1}^{* *}+\frac{U_{I, i+1}-U_{I, i+1}^{* *}}{C_{V, I}} \\
& B\left(T_{e, i+1}^{* * *}\right)=B\left(T_{e, i+1}^{* *}\right)+\left.\frac{\partial B_{v}}{\partial T}\right|_{T_{e, i+1}^{* *}}\left(T_{e, i+1}^{* * *}-T_{e, i+1}^{* *}\right)
\end{aligned}
$$

The host code then updates the final temperatures based on the final specific energies.

\subsection{Solution Methods}

Equations 30 to 32 are not easy to solve. We first simplify by substituting Equations 33 to 35 into Equations 30 to 32 , then solve Equation 31 for $T_{I, i+1}^{* * *}$ :

$$
T_{I, i+1}^{* * *}=h T_{e, i+1}^{* * *}+S_{I}
$$

where

$$
\begin{gathered}
g=\frac{\alpha \Delta t}{\rho C_{V, I}+\alpha \Delta t} \\
S_{I}=\frac{\rho C_{V, I} T_{I, i+1}^{* *}}{\rho C_{V, I}+\alpha \Delta t}
\end{gathered}
$$


We likewise solve for $T_{e, i+1}^{* * *}$ :

$$
T_{e, i+1}^{* * *}=h \int \sigma_{a, v, i} E_{v, i+1} d v+S_{T}
$$

where

$$
\begin{aligned}
& h=\frac{c \Delta t}{\rho C_{V, e}+\frac{\alpha \Delta t}{\rho C_{V, e}}(1-h)+\left.4 \pi \int \sigma_{a, v, i} \frac{\partial B_{v}}{\partial T}\right|_{T_{e, i+1}^{* *}} d v \Delta t} \\
& S_{T}=\frac{T_{e, i+1}^{* *}+\alpha S_{I}-4 \pi \int \sigma_{a, v, i}\left(B\left(T_{e, i+1}^{* *}\right)-\left.\frac{\partial B v}{\partial T}\right|_{T_{e, i+1}^{* *}} T_{e, i+1}^{* *}\right) d v \Delta t}{\rho C_{V, e}+\frac{\alpha \Delta t}{\rho C_{V, e}}(1-h)+\left.4 \pi \int \sigma_{a, v, i} \frac{\partial B_{v}}{\partial T}\right|_{T_{e, i+1}^{* *}} d v \Delta t}
\end{aligned}
$$

And, finally,

$$
\frac{I_{v, i+1}}{c \Delta t}+\hat{n} \cdot \nabla I_{v, i+1}+\sigma_{t, s, i} I_{v, i+1}=f_{v} \int \sigma_{a, v, i} E_{v, i+1} d v+\frac{c}{4 \pi} \sigma_{v, s, i} E_{v, i+1}+S_{v}
$$

where

$$
\begin{aligned}
& f_{v}=\left.h \sigma_{v, a, i} \frac{\partial B_{v}}{\partial T}\right|_{T_{e, i+1}^{* *}} \\
& S_{v}=\sigma_{v, a, i}\left(B\left(T_{e, i+1}^{* *}\right)+\left.\frac{\partial B_{v}}{\partial T}\right|_{T_{e, i+1}^{* *}}\left(S_{T}-T_{e, i+1}^{* *}\right)\right)+\frac{I_{v, i+1}^{* *}}{c \Delta t}
\end{aligned}
$$

We simplify further by using operator notation:

$$
L I_{i+1}=M \Sigma D I_{i+1}+S
$$

where $L$ is the advection-removal operator, $M$ is the moment to ordinate operator, $\Sigma$ is the scattering operator, $D$ is the ordinate to moment operator, and $S$ is the source. $M$ converts a moment representation of the radiation field, which for our purposes is almost always truncated at the zeroth moment, and converts it to an ordinate representation. $D$ converts the ordinte representation to moments. Thus $D M$ is the identity operator, though neither $D$ nor $M$ is usually square, let alone nonsingular.

Much of the effort on Serrano has focused on efficiently computing the inverse of $L$, which is called the sweep operator, since its solution requires "sweeping" the mesh along ordinate directions. Given a sweep solver, we then solve

$$
I_{i+1}=L^{-1}\left(M \Sigma D I_{i+1}+S\right)
$$

Several solution methods are provided by Serrano for solvine Equation 46 for different conditions.

Source Iteration. This method is useful in the streaming limit:

$$
I_{i+1}^{(k+1)}=L^{-1}\left(M \Sigma D I_{i+1}^{(k)}+S\right)
$$


That is, for the current iterate $\mathrm{I}_{\mathrm{i}+1}^{(\mathrm{k})}$, compute a source $M \Sigma D \mathrm{I}_{\mathrm{i}+1}^{(\mathrm{k})}+\mathrm{S}$ and then apply the sweep solver $L^{-1}$ to this source.

Source iteration performs well in the streaming limit, where $\Sigma$ is small. However, it performs increasingly poorly as one approaches the diffusion limit; although the method always converges, the spectral radius of the iteration scheme becomes arbitrarily close to unity. The primary value of source iteration is as a basis on which to build better schemes.

Krylov Solve. Krylov methods perform reasonably well for quite a wide range of problems. There are numerous such. methods, all based (at least in theory) on constructing a solution from the Krylov sequence

$$
a_{i+1}=A a_{i}
$$

where $a_{i}$ is the Krylov sequence and $A$ is the linear opertor to be inverted. For Serrano, $A=L^{-1} M \Sigma D$.

Linear Multifrequency-Gray Acceleration. Both source iteration and Krylov solution converge well if the problem is not strongly scattering. If the problem is strongly scattering, so that it approaches the diffusion limit, convergence can be improved using linear multifrequency-gray (LMFG) acceleration as a preconditioner.

This preconditioner is based on the observation that, if thermal reemission dominates, then the most slowly converging modes of the radiation field are the diffusive modes whose shape $\zeta_{V}$ is that of the reemission:

$$
\zeta_{v}=\frac{\frac{f_{v}}{\sigma_{v, t}+\frac{1}{c \Delta t}}}{\int \frac{f_{v}}{\sigma_{v, t}+\frac{1}{c \Delta t}} d v}
$$

For MFG-accelerated source iteration, we write

$$
I_{i+1}^{(k+1 / 2)}=L^{-1}\left(M \Sigma D I_{i+1}^{(k)}+S\right)
$$

that is, we perform a source iteration step, but label the result $k+1 / 2$ rather than $k+1$. We then solve the equation

$$
-\nabla \cdot D \nabla \delta \phi+\sigma \delta \phi=R
$$

where

$$
\begin{aligned}
& D=\frac{1}{3} \int \frac{\zeta_{v}}{\sigma_{v, t}+\frac{1}{c \Delta t}} d v \\
& \sigma=\int\left(\sigma_{v, t}-\sigma_{v, s}-\left(\frac{c}{4 \pi} \int f_{v} d v\right) \sigma_{a, v, i}\right) \zeta_{v} d v+\frac{1}{c \Delta t} \\
& R=\left(\frac{c}{4 \pi} \int f_{v} d v\right)\left(\int \sigma_{a, v, i}\left(E_{v}^{(k+1 / 2}-E_{v}^{(k)}\right) d v\right)
\end{aligned}
$$

Then 
(55) $\quad I_{v, i+1}^{(k+1)}=I_{v, i+1}^{(k+1 / 2)}+\zeta_{v} \delta \phi$

\section{References}

Lowrie, R.B., and Morel, J.E. 1998. Los Alamos National Laboratory Report LAUR-98-5332. 